\title{
ON CONVERGENCE OF THE IMMERSED BOUNDARY METHOD FOR ELLIPTIC INTERFACE PROBLEMS
}

\begin{abstract}
ZHILIN LI
Abstract. Peskin's Immersed Boundary (IB) method has been one of the most popular numerical methods for many years and has been applied to problems in mathematical biology, fluid mechanics, material sciences, and many other areas. Peskin's IB method is associated with discrete delta functions. It is believed that the IB method is first order accurate in the $L^{\infty}$ norm. But almost no rigorous proof could be found in the literature until recently [Mori, Comm. Pure. Appl. Math: 61:2008] in which the author showed that the velocity is indeed first order accurate for the Stokes equations with a periodic boundary condition. In this paper, we show first order convergence with a $\log h$ factor of the IB method for elliptic interface problems with Dirichlet boundary conditions.
\end{abstract}

\section{INTRODUCTION}

Since its introduction in 1970s, the Immersed Boundary (IB) method [16] has been applied to mathematics, engineering, biology, fluid mechanics, and many areas; see for example, 17] for a review and references therein. The IB method is not only a mathematical modeling tool in which a complicated boundary condition can be treated as a source distribution but also a numerical method in which a discrete delta function is used. The IB method is robust, simple, and has been applied to many problems.

It is widely believed that Peskin's IB method is only first order accurate in the $L^{\infty}$ norm. However, there was almost no rigorous proof in the literature until recently [15, in which the author has proved the first order accuracy of the IB method for the Stokes equations with a periodic boundary condition. The proof is based on some known inequalities between the fundamental solution and the discrete Green function with a periodic boundary condition for Stokes equations. In [4], the author showed that the pressure obtained from IB method has $O\left(h^{1 / 2}\right)$ order of convergence in the $L^{2}$ norm for a 1D model. In 20, 21, the authors designed some level set methods based on discrete delta functions. With suitable quadrature formulas in the integral form using the Green functions, the authors show that their approach can get the expected accuracy. However, there are few theoretical proofs on the IB method for elliptic interface problems with general boundary conditions. This is the main motivation of this paper. One difficulty is that there are little known estimates between the fundamental solution and the

Received by the editor January 26, 2012 and, in revised form, March 1, 2013.

2010 Mathematics Subject Classification. Primary 65N06, 65M12, 65M15.

Key words and phrases. Immersed Boundary (IB) method, Dirac delta function, convergence of IB method, discrete Green function, discrete Green's formula.

The author was supported in part by the AFSOR grant FA9550-09-1-0520, and the NIH grant 096195-01. 
discrete Green function with Dirichlet or other boundary conditions on rectangular domains. Compared with the case of periodic boundary conditions where there are existing estimates between the discrete Green function and the continuous one [8], there is almost none for Dirichlet and other boundary conditions. The main goal of this paper is to provide a convergence proof for the IB method for elliptic interface problems with Dirichlet boundary conditions. We will show that with commonly used discrete delta functions that satisfy the zeroth moment condition and first order interpolation property, the IB method is indeed first order convergent in the $L^{\infty}$ norm with a $\log h$ factor. The key in our proof is to establish a connection between the discrete Green function and the continuous one. Our proof is essentially independent of the boundary conditions and it is valid in $1 \mathrm{D}, 2 \mathrm{D}$, and $3 \mathrm{D}$ cases. The result should be applicable for many IB methods involving Stokes and NavierStokes solvers.

\section{Proof of the Convergence of the IB method in 1D}

We will give a proof for the 1D model,

$$
u^{\prime \prime}=c \delta(x-\alpha), \quad 0<x<1, \quad 0<\alpha<1, \quad u(0)=u(1)=0,
$$

in this section. Note that the analytic solution to the equation above is

$$
u(x)= \begin{cases}-c x(1-\alpha) & \text { if } x \leq \alpha, \\ -c \alpha(1-x) & \text { otherwise. }\end{cases}
$$

Given a uniform Cartesian grid $x_{i}=i h, i=0,1, \cdots, n, h=1 / n$, the IB method leads to the following system of linear equations,

$$
\frac{U_{i-1}-2 U_{i}+U_{i+1}}{h^{2}}=c \delta_{h}\left(x_{i}-\alpha\right), \quad i=1,2, \cdots, n-1,
$$

where $U_{i}$ is the finite difference approximation of the solution $u\left(x_{i}\right)$, and $\delta_{h}\left(x_{i}-\alpha\right)$ is a discrete delta function applied to the grid point $x_{i}$. In the matrix-vector form, the above finite difference equations can be written as $A_{h} \mathbf{U}=\mathbf{F}$, where $A_{h}$ is the tri-diagonal matrix formed by the discrete Laplacian. It is well known that $-A_{h}$ is a symmetric positive definite matrix (SPD) and diagonally dominant. Note that, a discrete delta function has compact support in the neighborhood of the interface $\alpha$, that is, $\delta_{h}(x) \neq 0$ only if $|x| \leq W h$, where $W$ is a constant. Commonly used discrete delta functions include the hat discrete delta function $\left(\delta^{h a t}(x)\right.$ with $\left.W=1\right)$ :

$$
\delta_{h}^{h a t}(x)= \begin{cases}(h-|x|) / h^{2}, & \text { if }|x|<h, \\ 0, & \text { if }|x| \geq h,\end{cases}
$$

and Peskin's original discrete cosine delta function $\left(\delta^{\text {cosine }}(x)\right.$ with $\left.W=2\right)$ :

$$
\delta_{h}(x)^{\text {cosine }}= \begin{cases}\frac{1}{4 h}(1+\cos (\pi x / 2 h)), & \text { if }|x|<2 h, \\ 0, & \text { if }|x| \geq 2 h ;\end{cases}
$$

see for example, [13. Note that, when we use the hat delta function, the result is the same as that of the IIM for the simple model. The solution to the finite difference equations is the same as the true solution if there are no round-off errors, that is,

(2.6) $\frac{u\left(x_{i-1}\right)-2 u\left(x_{i}\right)+u\left(x_{i+1}\right)}{h^{2}}=c \delta_{h}^{h a t}\left(x_{i}-\alpha\right), \quad i=1,2, \cdots, n-1 ;$ 
see for example, 3, 13. But this is not the case for other discrete delta functions.

We define the error vector as $\mathbf{E}=\left\{E_{i}\right\}$, where $E_{i}=u\left(x_{i}\right)-U_{i}$. We also define $\mathbf{u}=\left\{u\left(x_{i}\right)\right\}$. The local truncation error is defined as $\mathbf{T}=\left\{T_{i}\right\}$,

$$
T_{i}=\frac{u\left(x_{i-1}\right)-2 u\left(x_{i}\right)+u\left(x_{i+1}\right)}{h^{2}}-c \delta_{h}\left(x_{i}-\alpha\right) .
$$

With the definition, we have $A_{h} \mathbf{U}=\mathbf{F}, A_{h} \mathbf{u}=\mathbf{F}+\mathbf{T}$, and therefore $A_{h} \mathbf{E}=\mathbf{T}$. For the hat discrete delta function, we have $\left|T_{i}\right|=0$ for all $i$ 's for the simple model. For the cosine discrete delta function or other discrete delta functions, generally we have $\left|T_{j}\right|=O(1 / h)$ for a few grid points neighboring the interface $\alpha$; see for example Table 11 on page 1187. So one interesting question is: Why is the IB method still first order accurate, that is, $\|E\|_{\infty}=O(h)$ ? To answer this question, we first introduce the following lemma.

Lemma 2.1. Let $A_{h} \mathbf{y}=\mathbf{e}_{k}$ and $y_{0}=y_{n}=0$, where $\mathbf{e}_{k}$ is the $k$-th unit base vector, then

$$
y_{i}= \begin{cases}-h x_{i}\left(1-x_{k}\right) & \text { if } i \leq k, \\ -h x_{k}\left(1-x_{i}\right) & \text { otherwise. }\end{cases}
$$

The significance of this lemma is that the solution is order $h$ smaller than the concentrated source.

Proof. We note the following identity:

$$
A_{h} \mathbf{y}=h \frac{\mathbf{e}_{k}}{h}=h \delta_{h}^{h a t}(0) \mathbf{e}_{k} .
$$

For this simple case, the IB method using the hat discrete delta function is identical to the IIM; see [13. Thus from the Immersed Interface Method (see [3,13]) we know that $\mathbf{y}$ is the exact discrete solution at the grid points of the following boundary value problem

$$
u^{\prime \prime}=h \delta\left(x-x_{k}\right), \quad 0<x<1, \quad u(0)=u(1)=0,
$$

whose solution is

$$
y(x)= \begin{cases}-h x\left(1-x_{k}\right) & \text { if } x \leq x_{k}, \\ -h x_{k}(1-x) & \text { otherwise. }\end{cases}
$$

This completes the proof. Note that $|y(x)| \leq h$.

From this lemma, we have the following corollary.

Corollary 2.2. Let $A_{h} \mathbf{y}=\mathbf{r}$, then $|y(x)| \leq h W\|r\|_{\infty}$, where $W$ is the number of non-zero components of $\mathbf{r}$.

The proof is straightforward from (2.11) and the fact that $0 \leq x \leq 1$ and $0 \leq 1-x \leq 1$. Notice that for a discrete delta function, it should satisfy at least the zeroth moment equation (see [3]), that is,

$$
\sum_{i} \delta_{h}\left(x_{i}-\alpha\right)=1,
$$

corresponding to the continuous case $\int \delta_{h}(x-\alpha) d x=1$ if $\alpha$ is in the interval of the integration. Now we are ready to prove the main theorem. 
Theorem 2.3. Let $u(x)$ be the solution to (2.1) and $\mathbf{U}$ is the solution obtained from the immersed boundary method (2.3) using a discrete delta function $\delta_{h}(x)$ for (2.1). Then $\mathbf{U}$ is first order accurate, that is,

$$
\|\mathbf{E}\|_{\infty} \leq \bar{C} h
$$

where $\bar{C}$ is a constant.

Proof. We can decompose the local truncation error into two groups,

$$
\mathbf{T}=\mathbf{T}^{r e g}+\mathbf{T}^{i r r e g}
$$

where $\left\|\mathbf{T}_{r e g}\right\|_{\infty}=0$ corresponds to the local truncation errors at regular grid points where $\delta_{h}\left(x_{i}-\alpha\right)=0$ and the true solution is piecewise linear. Note that, we have

$$
\sum_{i=1}^{n-1} T_{i}=\sum \mathbf{T}^{r e g}+\sum \mathbf{T}^{i r r e g}=O+\sum \mathbf{T}^{i r r e g}
$$

On the other hand, we also have

$$
\frac{u\left(x_{i-1}\right)-2 u\left(x_{i}\right)+u\left(x_{i+1}\right)}{h^{2}}=c \delta_{h}^{h a t}\left(x_{i}-\alpha\right)
$$

since the finite difference method using the discrete delta function gives the exact solution at all the grid points. Thus we have

$$
\begin{aligned}
\sum_{i=1}^{n-1} T_{i} & =\sum_{i=1}^{n-1}\left(\frac{u\left(x_{i-1}\right)-2 u\left(x_{i}\right)+u\left(x_{i+1}\right)}{h^{2}}-c \delta_{h}\left(x_{i}-\alpha\right)\right) \\
& =\sum_{i=1}^{n-1} c \delta_{h}^{h a t}\left(x_{i}-\alpha\right)-\sum_{i=1}^{n-1} c \delta_{h}\left(x_{i}-\alpha\right)=0,
\end{aligned}
$$

from the zeroth moment equation (2.12). Thus we have $\sum_{i} T_{i}^{\text {irreg }}=0$. We divide $T_{i}^{\text {irreg }}$ into two groups, one with all positive $T_{i}$ 's denoted as $T_{i}^{\text {irreg }{ }^{+}+}$; the other one with all the negative $T_{i}$ 's denoted as $T_{i}^{\text {irreg,- }}$. Since $\sum T_{i}^{\text {irreg, }+}+\sum T_{i}^{\text {irreg, }-}=0$, $T_{i}^{\text {irreg, }+}$ and $T_{i}^{\text {irreg,- }}$ must have the same order of the magnitude $O(1 / h)$ although those index $i$ are different except that $\left|x_{i}-\alpha\right| \leq W h$ is true for all irregular grid points. Because the solution is linear with $c$, we have

$$
\mathbf{E}=A_{h}^{-1} \mathbf{T}
$$


From the solution expression, we know that,

$$
\begin{aligned}
& E_{l}=\left(A_{h}^{-1} \mathbf{T}\right)_{l}=-\left(\sum_{x_{l} \leq x_{i}} T_{i}^{i r r e g} x_{l}\left(1-x_{i}\right)+\sum_{x_{l}>x_{i}} T_{j}^{i r r e g} x_{i}\left(1-x_{l}\right)\right) \\
& = \begin{cases}-x_{l} \sum_{x_{l} \leq x_{i}} T_{i}^{\text {irreg }}\left(1-x_{i}\right) & \text { if } x_{l} \leq \min _{i} x_{i}^{\text {irreg }}, \\
-\left(1-x_{l}\right) \sum_{x_{l}>x_{i}} T_{j}^{i r r e g} x_{i} & \text { if } x_{l}>\min _{i} x_{i}^{\text {irreg }}, \\
-\left(x_{l} \sum_{x_{l} \leq x_{i}} T_{i}^{i r r e g}\left(1-x_{i}\right)+\left(1-x_{l}\right) \sum_{x_{l}>x_{i}} T_{j}^{\text {irreg }} x_{i}\right) & \text { otherwise }\end{cases} \\
& =\left\{\begin{array}{lc}
-x_{l} \sum_{x_{l} \leq x_{i}} T_{i}^{\text {irreg }}(1-\alpha)+O(W h) & \text { if } x_{l} \leq \min _{i} x_{i}^{\text {irreg }}, \\
-\left(1-x_{l}\right) \sum_{x_{l}>x_{i}} T_{j}^{\text {irreg }} \alpha+O(W h) & \text { if } x_{l}>\min _{i} x_{i}^{\text {irreg }}, \\
-\left(\sum_{x_{l} \leq x_{i}} T_{i}^{\text {irreg }} \alpha(1-\alpha)+\sum_{x_{l}>x_{i}} T_{j}^{\text {irreg }} \alpha(1-\alpha)\right)+O(W h) & \text { otherwise }
\end{array}\right. \\
& =0+O(W h) \text {, }
\end{aligned}
$$

after we expand all $x_{i}$ 's at $\alpha$ and since all related $x_{i}$ and $x_{j}$ are within $W h$ distance from the interface $\alpha$. This completes the proof.

\section{Proof of the Convergence of the IB method in 2D}

The discussion for 2D problems is much more challenging since the interface is often a curve instead of a point. In [15], the author has proved the first order convergence of the IB method for the Stokes equations with a periodic boundary condition in 2D based on existing estimates between the discrete Green function and the continuous one in [8]. However, there are almost no theoretical proofs on the IB method for elliptic interface problems or other PDEs with general boundary conditions. We will prove that the result obtained from the IB for the elliptic interface problem with a Dirichlet boundary condition is indeed first order accurate in this section.

Consider the following 2D elliptic interface problem,

$$
\begin{aligned}
& \Delta u(x, y)=f(x, y)+\int_{\Gamma} v(s) \delta(x-X(s))(y-Y(s)) d s, \quad(x, y) \in \Omega, \\
& \left.u(x, y)\right|_{\partial \Omega}=u_{0}(x, y)
\end{aligned}
$$

where we assume that $f(x, y) \in C(\Omega), \Gamma \in C^{1}, v(s) \in C^{1}$. Without loss of generality, we assume that $\Omega$ is a unit square $0 \leq x, y \leq 1$, see Figure 1 for an illustration. The problem can be decomposed as the sum of the solutions of the following two problems. The first one is

$$
\begin{aligned}
& \Delta u_{1}(x, y)=f(x, y), \\
& \left.u_{1}(x, y)\right|_{\partial \Omega}=u_{0}(x, y),
\end{aligned}
$$




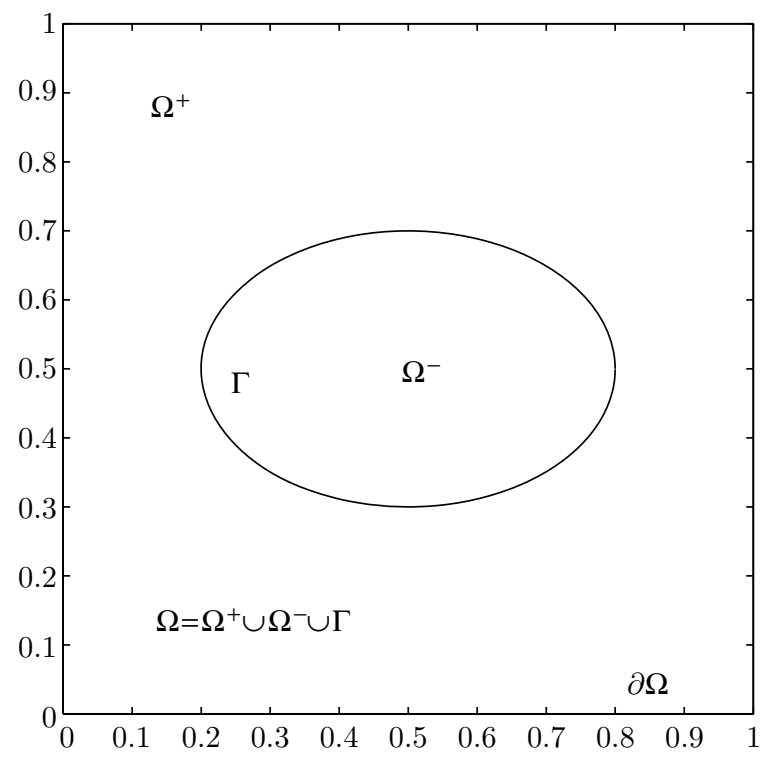

Figure 1. A diagram of a $2 \mathrm{D}$ elliptic interface problem. The interface is $\Gamma$.

which is a regular problem whose solution is smooth, that is, $u_{1}(x, y) \in C^{2}(\Omega)$. The second problem is

$$
\begin{aligned}
& \Delta u_{2}(x, y)=\int_{\Gamma} v(s) \delta(x-X(s))(y-Y(s)) d s, \\
& \left.u_{2}(x, y)\right|_{\partial \Omega}=0 .
\end{aligned}
$$

The solution to the second problem is equivalent to the following problem,

$$
\begin{aligned}
& \Delta u_{2}(x, y)=0, \quad\left[u_{2}\right]_{\Gamma}=0, \quad\left[\frac{\partial u_{2}}{\partial n}\right]_{\Gamma}=v(s), \\
& \left.u_{2}(x, y)\right|_{\partial \Omega}=0 .
\end{aligned}
$$

The solution to the original problem is $u=u_{1}+u_{2}$. Since $u_{1}$ is the solution to a regular problem, it is enough just to consider $u_{2}(x, y)$. Thus we will simply use the notation $u(x, y)$ for $u_{2}(x, y)$.

Peskin's IB method for the problem includes the following steps:

- Generate a uniform Cartesian mesh $x_{i}=i h, y_{j}=j h, i, j=0,1, \cdots, n$. Here we use a uniform mesh for simplicity. We denote $\Omega_{h}$ as the set of all grid points; and $\partial \Omega_{h}$ as the grid points on the boundary.

- Replace the partial derivatives with the finite difference approximation and use a discrete delta function to spread the singular source to nearby grid points, that is, for $i, j=1,2, \cdots, n-1$,

$$
\begin{aligned}
& \frac{U_{i-1, j}+U_{i+1, j}+U_{i, j-1}+U_{i, j+1}-4 U_{i j}}{h^{2}}=C_{i j}^{I B}, \\
& C_{i j}^{I B}=\sum_{k=1}^{N_{b}} v_{k} \delta_{h}\left(x_{i}-X_{k}\right) \delta_{h}\left(y_{j}-Y_{k}\right) \Delta s_{k},
\end{aligned}
$$


where $\left(X_{k}, Y_{k}\right), k=1,2, \cdots, N_{b}$, is a discretization of the interface $\Gamma$, and $v_{k} \approx v\left(s_{k}\right)$, which we assume it is at least first order approximation, $v_{k}=v\left(s_{k}\right)+O(h)$.

- Solve the finite difference system of equations above to get an approximation solution $\left\{U_{i j}\right\}$. This can be done by calling a fast Poisson solver, say [1].

3.1. Discrete delta functions and discrete Green functions. As a common practice, we assume that $\max _{k}\left\{\Delta s_{k}\right\}=\Delta s \sim O(h)$. In Peksin's IB method, a discrete delta function is used for two purposes. One is to spread the singular source to the nearby grids. The other one is to interpolate a grid function, say the velocity, to get its values on the interface. Thus the discrete delta function used should satisfy at least the zeroth moment condition as described in [3]. The interpolation using the discrete delta function should be at least first order accurate, that is,

$$
\sum_{i, j=1}^{n-1} h^{2} \sum_{k=1}^{N_{b}} v_{k} \delta_{h}\left(x_{i}-X_{k}\right) \delta_{h}\left(y_{j}-Y_{k}\right) \Delta s_{k}=\int_{\Gamma} v(s) d s+O(h),
$$

which corresponds to

$$
\iint_{\Omega} \int_{\Gamma} v(s) \delta(x-X(s)) \delta(y-Y(s)) d s d x d y=\int_{\Gamma} v(s) d s .
$$

From $\iint_{\Omega} u(x, y) \delta(x-X) \delta(y-Y) d x d y=u(X, Y)$, we should also have the interpolation property,

$$
\sum_{i, j=1}^{n-1} h^{2} u\left(x_{i}, y_{j}\right) \delta_{h}\left(x_{i}-X_{k}\right) \delta_{h}\left(y_{j}-Y_{k}\right)=u(X, Y)+O(h) .
$$

In (3.6) and (3.8), the error terms depend on the first order derivatives of $v(s)$ and $u(x, y)$, respectively.

A discrete delta function has a compact support, that is,

$$
\begin{aligned}
& \delta_{h}\left(x_{i}-X_{k}\right)=0, \quad \text { if }\left|x_{i}-X_{k}\right|>W h, \\
& \quad \text { and } \quad \delta_{h}\left(y_{j}-Y_{k}\right)=0, \quad \text { if }\left|y_{j}-Y_{k}\right|>W h,
\end{aligned}
$$

where $\mathbf{x}_{i j}=\left(x_{i}, y_{j}\right)$, and $W$ is a constant.

We define the error vector as $\mathbf{E}=\left\{E_{i j}\right\}$, where $E_{i j}=u\left(x_{i}, y_{j}\right)-U_{i j}$. We also define $\mathbf{U}=\left\{u\left(x_{i}, y_{j}\right)\right\}$. The local truncation error is defined as $\mathbf{T}=\left\{T_{i j}\right\}$,

$$
T_{i j}=\frac{u\left(x_{i-1}, y_{j}\right)+u\left(x_{i+1}, y_{j}\right)+u\left(x_{i}, y_{j-1}\right)+u\left(x_{i}, y_{j+1}\right)-4 u\left(x_{i}, y_{j}\right)}{h^{2}}-C_{i j}^{I B} \text {. }
$$

In the matrix vector form, we have $A_{h} \mathbf{U}=\mathbf{F}, A_{h} \mathbf{u}=\mathbf{F}+\mathbf{T}$, and therefore $A_{h} \mathbf{E}=$ $\mathbf{T}$, where $A_{h}$ is the matrix formed by the discrete Laplacian. We have $\left|T_{i j}\right|=O\left(h^{2}\right)$ at regular grid points where $C_{i j}^{I B}=0$. In general, we have $\left|T_{i j}\right|=O(1 / h)$ for grid points neighboring the interface $\Gamma$ except for the correction terms using the Immersed Interface Method (IIM) [1] 13] for which we have $\left|T_{i j}^{I I M}\right|=O(h)$ at irregular grid points where the interface cuts through the standard five-point stencil.

It is interesting that the local truncation errors can have order $O(1 / h)$ at some grid points, but the global error is still of $O(h)$. There has to be some kind of cancelations of the errors, which can be seen from our proof process. 
Definition 3.1. Let $\mathbf{e}_{l m}$ be the unit grid function whose values are zero at all grid points except at $\mathbf{x}_{l m}=\left(x_{l}, y_{m}\right)$ where its component is $e_{l m}=1$. The discrete Green function centered at $\mathbf{x}_{l m}$ with homogeneous boundary condition is defined as

$$
\mathbf{G}^{h}\left(\mathbf{x}_{i j}, \mathbf{x}_{l m}\right)=\left(A_{h}^{-1} \mathbf{e}_{l m} \frac{1}{h^{2}}\right)_{i j}, \quad \mathbf{G}^{h}\left(\partial \Omega_{h}, \mathbf{x}_{l m}\right)=0
$$

where $\partial \Omega_{h}$ denotes the grid points on the boundary $\partial \Omega$.

Note that from Remark 4.4.6 in [7, we know that $G_{h}\left(\mathbf{x}_{i j}, \mathbf{x}_{l m}\right)$ is symmetric,

$$
G^{h}\left(\mathbf{x}_{i j}, \mathbf{x}_{l m}\right)=G^{h}\left(\mathbf{x}_{l m}, \mathbf{x}_{i j}\right)
$$

The usual discrete Green function, also called a discrete fundamental solution, on the entire integer lattice is defined as

$$
\Delta_{h} g_{h}\left(\mathbf{x}_{i j}, \mathbf{x}_{l m}\right)= \begin{cases}\frac{1}{h^{2}}, & \text { if } \mathbf{x}_{i j}=\mathbf{x}_{l m} \\ 0, & \text { otherwise }\end{cases}
$$

for all integers $i$ and $j$; see, for example, [2, 6, 7, 10, 15, 18, 19] for more discussions about the discrete Green's functions. Note that $g_{h}\left(\mathbf{x}_{i j}, \mathbf{x}_{l m}\right)$ is also symmetric, that is, $g_{h}\left(\mathbf{x}_{i j}, \mathbf{x}_{l m}\right)=g_{h}\left(\mathbf{x}_{l m}, \mathbf{x}_{i j}\right)$.

To prepare the convergence proof, we first list some lemmas that are either directly or indirectly used in the convergence proof. The following lemma is the discrete first Green formula. Although it is not directly used in the proof, it shows how the discrete summation is related to the integral form; and how the source distribution is related to the jump conditions.

Lemma 3.2 (The discrete first Green formula and an error estimate). Let $u(x, y)$ be the solution to (3.1). Thus $u(x, y)$ is in the piecewise $C^{1}(\Omega)$ space, that is, $u(x, y) \in C^{1}(\Omega \backslash \Gamma)$. Assuming that the distance between $\Gamma$ and $\partial \Omega$ is $O(1)$, that is, $\operatorname{dist}(\Gamma, \partial \Omega) \sim O(1)$, then we have

$$
\sum_{i, j=1}^{n-1} \Delta_{h} u\left(x_{i}, y_{j}\right) h^{2}=\int_{\partial \Omega} \frac{\partial u}{\partial n} d s+O(h)=\int_{\Gamma} v(s) d s+O(h),
$$

where

$$
\Delta_{h} u\left(x_{i}, y_{j}\right)=\frac{u\left(x_{i-1}, y_{j}\right)+u\left(x_{i+1}, y_{j}\right)+u\left(x_{i}, y_{j-1}\right)+u\left(x_{i}, y_{j+1}\right)-4 u\left(x_{i}, y_{j}\right)}{h^{2}},
$$

is the discrete Laplacian using the standard five-point stencil, and the summation is over all the interior grid points.

Proof. We first prove the discrete first Green formula by expanding the summation. After cancellation of interior terms, only boundary terms are left in the summation 
as follows:

$$
\begin{aligned}
\sum_{i j} \Delta_{h} u\left(x_{i}, y_{j}\right) h^{2}= & \sum_{j=1}^{n-1} h \frac{u\left(x_{0}, y_{j}\right)-u\left(x_{1}, y_{j}\right)}{h}+\sum_{j=1}^{n-1} h \frac{u\left(x_{n}, y_{j}\right)-u\left(x_{n-1}, y_{j}\right)}{h} \\
& +\sum_{i=1}^{n-1} h \frac{u\left(x_{i}, y_{0}\right)-u\left(x_{i}, y_{1}\right)}{h}+\sum_{i=1}^{n-1} h \frac{u\left(x_{i}, y_{n}\right)-u\left(x_{i}, y_{n-1}\right)}{h} \\
= & \int_{\partial \Omega} \frac{\partial u}{\partial n} d s+O(h) .
\end{aligned}
$$

On the other hand, by integrating both sides of the partial differential equation (3.1) with $f(x, y)=0$ and $u_{0}(x, y)=0$, we get

$$
\iint_{\Omega} \Delta u d x d y=\iint_{\Omega}\left(\int_{\Gamma} v(s) \delta(x-X(s))(y-Y(s)) d s\right) d x d y
$$

or equivalently,

$$
\int_{\partial \Omega} \frac{\partial u}{\partial n} d s=\int_{\Gamma} v(s) d s
$$

This completes the proof.

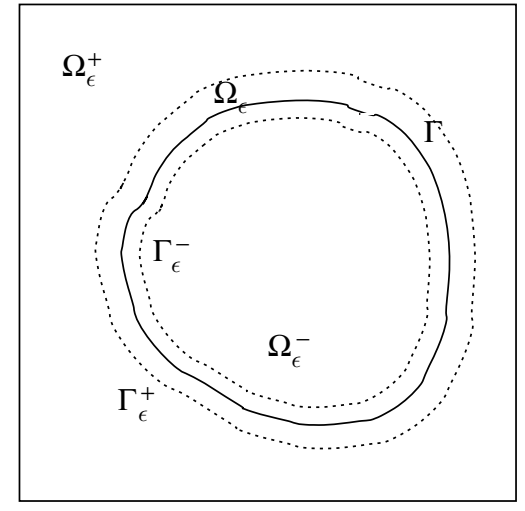

FiguRE 2. A diagram of the domain, interface, and integration.

Remark 3.3. The double integral $\iint_{\Omega} \Delta u d x d y$ can be divided into three parts

$$
\begin{aligned}
\iint_{\Omega} \Delta u d x d y & =\iint_{\Omega_{\epsilon}^{+}} \Delta u d x d y+\iint_{\Omega_{\epsilon}^{-}} \Delta u d x d y+\iint_{\Omega_{\epsilon}} \Delta u d x d y \\
& =\int_{\partial \Omega} \frac{\partial u}{\partial n} d s-\int_{\Gamma_{\epsilon}^{+}} \frac{\partial u}{\partial n} d s+\int_{\Gamma_{\epsilon}^{+}} \frac{\partial u}{\partial n} d s
\end{aligned}
$$

see Figure 2 for an illustration, see also [12. As $\epsilon \rightarrow 0$, we have

$$
\lim _{\epsilon \rightarrow 0} \iint_{\Omega_{\epsilon}} \Delta u d x d y=\int_{\Gamma} v(s) d s=\int_{\Gamma}\left[\frac{\partial u}{\partial n}\right] d s
$$


from the partial differential equation (3.1) with $f(x, y)=0$ and $u_{0}(x, y)=0$. Thus we get

$$
\lim _{\epsilon \rightarrow 0} \iint_{\Omega_{\epsilon}} \Delta u d x d y=\int_{\partial \Omega} \frac{\partial u}{\partial n} d s .
$$

While Lemma 3.2 is not directly used in our convergence proof, it is easier to illustrate the relation between the boundary integral along $\partial \Omega$ and the source distribution along $\Gamma$ for the first Green formula than for the second Green formula that is used in the proof.

3.2. Interpolating the discrete delta function. We know that $\mathbf{G}^{h}\left(\mathbf{x}_{i j}, \mathbf{x}_{l m}\right)$ defined in (3.10) is a grid function with a homogeneous Dirichlet boundary condition at the boundary grid points. The discrete Laplacian $\Delta_{h} \mathbf{G}^{h}\left(\mathbf{x}_{i j}, \mathbf{x}_{l m}\right)$ is zero at all interior grid points except that it is $1 / h^{2}$ at $\mathbf{x}_{l m}$. We can interpolate $\mathbf{G}^{h}\left(\mathbf{x}_{i j}, \mathbf{x}_{l m}\right)$ to the entire domain to get an interpolation function $\mathbf{G}_{I}^{h}\left(\mathbf{x}, \mathbf{x}_{l m}\right)$. We consider any such an interpolation function that satisfies the following:

- $\mathbf{G}_{I}^{h}\left(\mathbf{x}_{i j}, \mathbf{x}_{l m}\right)=\mathbf{G}^{h}\left(\mathbf{x}_{i j}, \mathbf{x}_{l m}\right)$.

- $\mathbf{G}_{I}^{h}\left(\mathbf{x}, \mathbf{x}_{l m}\right) \in C^{1}(\Omega) \cap H^{2}(\Omega)$.

- $\Delta_{h} \mathbf{G}_{I}^{h}\left(\mathbf{x}_{i j}, \mathbf{x}_{l m}\right)=\Delta_{h} \mathbf{G}^{h}\left(\mathbf{x}_{i j}, \mathbf{x}_{l m}\right)=0$, that is, zero for all $i$ and $j$ except for $i=l$ and $j=m$.

- The interpolation is second or higher order accurate, that is,

$$
\left|\partial^{\alpha} \mathbf{G}^{h}\left(\mathbf{x}_{i j}, \mathbf{x}_{l m}\right)-\partial^{\alpha} \mathbf{G}_{I}^{h}\left(\mathbf{x}_{i j}, \mathbf{x}_{l m}\right)\right| \leq C h^{3-\|\boldsymbol{\alpha}\|_{1}}, \quad \text { for } \quad\|\boldsymbol{\alpha}\|_{1} \leq 2,
$$

where the derivatives of $\mathbf{G}^{h}\left(\mathbf{x}_{i j}, \mathbf{x}_{l m}\right)$ is defined from finite differences (see [19]) and $\alpha$ is the summation notation as used in the literature for the Sobolev spaces.

- $\iint_{R_{l m}} \Delta \mathbf{G}_{I}^{h}\left(\mathbf{x}, \mathbf{x}_{l m}\right) d x d y=O(h)$ except for the four neighboring squares centered at $\mathbf{x}_{l m}$ on which $\iint_{R_{l m}} \Delta \mathbf{G}_{I}^{h}\left(\mathbf{x}, \mathbf{x}_{l m}\right) d x d y=1+O(h)$, where $R_{l m}$ is the square $\left[x_{l}-h, x_{l}+h\right] \times\left[y_{m}-h, y_{m}+h\right]$ and so on.

We provide a construction of such an interpolation function in Appendix A.

Lemma 3.4. Let $\mathbf{G}_{I}^{h}\left(\mathbf{x}, \mathbf{x}_{l m}\right)$ be an interpolation function of $\mathbf{G}^{h}\left(\mathbf{x}_{i j}, \mathbf{x}_{l m}\right)$ that satisfies the conditions above, then we have the following estimates:

$$
\begin{aligned}
& \mathbf{G}_{I}^{h}\left(\mathbf{x}, \mathbf{x}_{l m}\right) \leq \frac{1}{4}+\frac{1}{16} \log \left(\left\|\mathbf{x}-\mathbf{x}_{l m}\right\|_{2}^{2}+h^{2}\right)+O(h), \\
& \left|\partial^{\alpha} \mathbf{G}_{I}^{h}\left(\mathbf{x}, \mathbf{x}_{l m}\right)\right| \leq \frac{C}{\left(\left\|\mathbf{x}-\mathbf{x}_{l m}\right\|_{2}+h\right)^{\|\boldsymbol{\alpha}\|_{1}}}+O(h), \quad \text { if }\|\boldsymbol{\alpha}\|_{1} \leq k-1,
\end{aligned}
$$

where $k$ is the order of interpolation and $C$ is a constant. The inequality (3.16) is true if $\operatorname{dist}(\mathbf{x}, \partial \Omega) \sim O(1)$ and $\operatorname{dist}\left(\partial \Omega, \mathbf{x}_{l m}\right) \sim O(1)$.

Proof. From Remark 4.4.8 in [7, we know that

$$
\mathbf{G}^{h}\left(\mathbf{x}_{i j}, \mathbf{x}_{l m}\right) \leq \frac{1}{4}+\frac{1}{16} \log \left(\left\|\mathbf{x}_{i j}-\mathbf{x}_{l m}\right\|_{2}^{2}+h^{2}\right) .
$$

Thus from the order requirement of the interpolation function, we have the first inequality. To prove the second inequality, we use the expression from (4.11) in [18]:

$$
\mathbf{G}^{h}\left(\mathbf{x}_{i j}, \mathbf{x}_{l m}\right)=g_{h}\left(\mathbf{x}_{i j}, \mathbf{x}_{l m}\right)-\hat{s}\left(\mathbf{x}_{i j}, \mathbf{x}_{l m}\right)-s\left(\mathbf{x}_{i j}, \mathbf{x}_{l m}\right),
$$


where $\hat{s}\left(\mathbf{x}_{i j}, \mathbf{x}_{l m}\right)$ is chosen such that $\Delta_{h} \mathbf{G}^{h}\left(\mathbf{x}_{i j}, \mathbf{x}_{l m}\right)=0$ at the grid points on the boundary of the unit square using the method of images. Thus $\hat{s}\left(\mathbf{x}_{i j}, \mathbf{x}_{l m}\right)$ is a combination of $g_{h}\left(\mathbf{x}_{i j}, \mathbf{x}_{l m}\right)$ at points outside of the unit square. From Theorem 3.1 in [19], we have $\left|\partial^{\alpha} g_{h}\left(\mathbf{x}_{i j}, \mathbf{x}_{l m}\right)\right| \leq C /\left(\left\|\mathbf{x}_{i j}-\mathbf{x}_{l m}\right\|_{2}+h\right)^{\|\boldsymbol{\alpha}\|_{1}}$. The same can be said for $\hat{s}\left(\mathbf{x}_{i j}, \mathbf{x}_{l m}\right)$. The term $s\left(\mathbf{x}_{i j}, \mathbf{x}_{l m}\right)$ is chosen such that $\mathbf{G}^{h}\left(\mathbf{x}_{i j}, \mathbf{x}_{l m}\right)=0$ at the grid points on the boundary of the unit square and $\Delta_{h} s\left(\mathbf{x}_{i j}, \mathbf{x}_{l m}\right)=0$ at all other grid points. Thus $\left.s\left(\mathbf{x}_{i j}, \mathbf{x}_{l m}\right)\right|_{\partial \Omega_{h}}$ is the trace of $g_{h}\left(\mathbf{x}_{i j}, \mathbf{x}_{l m}\right)-\hat{s}\left(\mathbf{x}_{i j}, \mathbf{x}_{l m}\right)$; see Remark on page 297 in [5]. Since the values of $\left.s\left(\mathbf{x}_{i j}, \mathbf{x}_{l m}\right)\right|_{\partial \Omega_{h}}$ are from the discrete Laplacian, it can be smoothly extended to the entire boundary $\partial \Omega$. Thus from Lemma 2.7.5 in [9] and the maximum principle, we have

$$
\left|\partial^{\alpha} s\left(\mathbf{x}, \mathbf{x}_{l m}\right)\right| \leq\left(\frac{C\|\boldsymbol{\alpha}\|_{1}}{\operatorname{dist}(\mathbf{x}, \partial \Omega)}\right)^{\|\boldsymbol{\alpha}\|_{1}} \sup _{\Omega}|s|=\left(\frac{C\|\boldsymbol{\alpha}\|_{1}}{\operatorname{dist}(\mathbf{x}, \partial \Omega)}\right)^{\|\boldsymbol{\alpha}\|_{1}} \sup _{\partial \Omega}|s|
$$

where $C$ is another constant. From Section 4.4 in [18, we know that $s\left(\mathbf{x}_{i j}, \mathbf{x}_{l m}\right)=$ $O(1)$ as long as $\operatorname{dist}\left(\partial \Omega, \mathbf{x}_{l m}\right)=O(1)$. Thus as long as $\operatorname{dist}(\mathbf{x}, \partial \Omega) \sim O(1)$, we have $\left|\partial^{\alpha} s\left(\mathbf{x}, \mathbf{x}_{l m}\right)\right| \sim O(1)$. This completes the proof of the lemma.

Remark 3.5. The interpolation function is not unique. Along the boundary $\partial \Omega$, from the requirement of the interpolation function, $\frac{\partial}{\partial n} \mathbf{G}_{I}^{h}\left(\mathbf{x}_{i j}, \mathbf{x}_{l m}\right)$ does exist and is continuous. We have, for example,

$$
\frac{\partial \mathbf{G}_{I}^{h}\left(\mathbf{x}_{i j}, \mathbf{x}_{l m}\right)}{\partial x}=\frac{\mathbf{G}_{I}^{h}\left(\mathbf{x}_{1 j}, \mathbf{x}_{l m}\right)-\mathbf{G}_{I}^{h}\left(\mathbf{x}_{0 j}, \mathbf{x}_{l m}\right)}{h}+O(h)
$$

along the boundary $x=0$.

Using a similar procedure in proving the discrete Green formula, we can get the second discrete Green formula.

Lemma 3.6. Let $u(x, y)$ be the solution to (3.1) and $\mathbf{G}_{I}^{h}\left(\mathbf{x}, \mathbf{x}_{l m}\right)$ be an interpolation function of $\mathbf{G}^{h}\left(\mathbf{x}_{i j}, \mathbf{x}_{l m}\right)$ that satisfies the conditions listed in Section 3.2. If $l, m \neq$ 1 or $n-1$, then we have,

$$
\sum_{i j} \Delta_{h} u\left(x_{i}, y_{j}\right) \mathbf{G}^{h}\left(\mathbf{x}_{i j}, \mathbf{x}_{l m}\right) h^{2}=\int_{\Gamma} v(s) \mathbf{G}_{I}^{h}\left(\mathbf{X}(s), \mathbf{x}_{l m}\right) d s+O(h) .
$$

Proof. Again, we show the second discrete Green formula by expanding the summation. After cancellation of interior terms, only boundary terms and a source are 
left. Thus, we get

$$
\begin{aligned}
\sum_{i, j=1}^{n-1} & \Delta_{h} u\left(x_{i}, y_{j}\right) \mathbf{G}^{h}\left(\mathbf{x}_{i j}, \mathbf{x}_{l m}\right) h^{2}=\sum_{j=1}^{n-1} h \frac{u\left(x_{0}, y_{j}\right)-u\left(x_{1}, y_{j}\right)}{h} \mathbf{G}^{h}\left(\mathbf{x}_{1 j}, \mathbf{x}_{l m}\right) \\
& +\sum_{j=1}^{n-1} h \frac{u\left(x_{n}, y_{j}\right)-u\left(x_{n-1}, y_{j}\right)}{h} \mathbf{G}^{h}\left(\mathbf{x}_{n-1, j}, \mathbf{x}_{l m}\right) \\
& +\sum_{i=1}^{n-1} h \frac{u\left(x_{i}, y_{0}\right)-u\left(x_{i}, y_{1}\right)}{h} \mathbf{G}^{h}\left(\mathbf{x}_{i 1}, \mathbf{x}_{l m}\right) \\
& +\sum_{i=1}^{n-1} h \frac{u\left(x_{i}, y_{n}\right)-u\left(x_{i}, y_{n-1}\right)}{h} \mathbf{G}^{h}\left(\mathbf{x}_{i, n-1}, \mathbf{x}_{l m}\right) \\
& -\sum_{j=1}^{n-1} h \frac{\mathbf{G}^{h}\left(\mathbf{x}_{0 j}, \mathbf{x}_{l m}\right)-\mathbf{G}^{h}\left(\mathbf{x}_{1 j}, \mathbf{x}_{l m}\right)}{h} u\left(x_{1}, y_{j}\right) \\
& -\sum_{j=1}^{n-1} h \frac{\mathbf{G}^{h}\left(\mathbf{x}_{n, j}, \mathbf{x}_{l m}\right)-\mathbf{G}^{h}\left(\mathbf{x}_{n-1, j}, \mathbf{x}_{l m}\right)}{h} u\left(x_{n-1}, y_{j}\right) \\
& -\sum_{i=1}^{n-1} h \frac{\mathbf{G}^{h}\left(\mathbf{x}_{i 0}, \mathbf{x}_{l m}\right)-\mathbf{G}^{h}\left(\mathbf{x}_{i 1}, \mathbf{x}_{l m}\right)}{h} u\left(x_{i}, y_{1}\right) \\
& -\sum_{i=1}^{n-1} h \frac{\mathbf{G}^{h}\left(\mathbf{x}_{i, n}, \mathbf{x}_{l m}\right)-\mathbf{G}^{h}\left(\mathbf{x}_{i, n-1}, \mathbf{x}_{l m}\right)}{h} u\left(x_{i}, y_{n-1}\right) \\
& +\sum_{i, j=1}^{n-1} u\left(x_{i}, y_{j}\right) \Delta_{h} \mathbf{G}^{h}\left(\mathbf{x}_{i j}, \mathbf{x}_{l m}\right) h^{2} \\
& \left.\int_{\partial}(\mathbf{x}) \mathbf{G}_{I}^{h}\left(\mathbf{x}, \mathbf{x}_{l m}\right)-\frac{\partial \mathbf{G}_{I}^{h}\left(\mathbf{x}, \mathbf{x}_{l m}\right)}{\partial n} u(\mathbf{x})\right) d s+u\left(\mathbf{x}_{l m}\right)+O(h) .
\end{aligned}
$$

On the other hand, by integrating both sides of the partial differential equation (3.1) with $f=0$ and $u_{0}=0$, we get

$$
\iint_{\Omega} \mathbf{G}_{I}^{h}\left(\mathbf{x}, \mathbf{x}_{l m}\right) \Delta u d x d y=\iint_{\Omega}\left(\int_{\Gamma} v(s) \delta(x-X(s))(y-Y(s)) d s\right) \mathbf{G}_{I}^{h}\left(\mathbf{x}, \mathbf{x}_{l m}\right) d x d y
$$

or equivalently,

$$
\begin{aligned}
\int_{\partial \Omega} & \left(\frac{\partial u}{\partial n} \mathbf{G}_{I}^{h}\left(\mathbf{x}, \mathbf{x}_{l m}\right)-\frac{\partial \mathbf{G}_{I}^{h}\left(\mathbf{x}, \mathbf{x}_{l m}\right)}{\partial n} u\right) d s+\iint_{\Omega} u \Delta \mathbf{G}_{I}^{h}\left(\mathbf{x}, \mathbf{x}_{l m}\right) d x d y \\
= & \int_{\Gamma} v(s) \mathbf{G}_{I}^{h}\left(\mathbf{X}(\mathbf{s}), \mathbf{x}_{l m}\right) d s
\end{aligned}
$$


Note that

$$
\begin{aligned}
& \iint_{\Omega} u(\mathbf{x}) \Delta \mathbf{G}_{I}^{h}\left(\mathbf{x}, \mathbf{x}_{l m}\right) d x d y=\sum_{R_{i j} \backslash R_{l m}} \iint_{\Omega} u(\mathbf{x}) \Delta \mathbf{G}_{I}^{h}\left(\mathbf{x}, \mathbf{x}_{l m}\right) d x d y \\
& \quad+\iint_{R_{l m}} u(\mathbf{x}) \Delta \mathbf{G}_{I}^{h}\left(\mathbf{x}, \mathbf{x}_{l m}\right) d x d y \\
& =\sum_{R_{i j} \backslash R_{l m}} \iint_{R_{i j}}\left(u\left(\mathbf{x}_{i j}\right)+O(h)\right) O(h) d x d y \\
& \quad+\iint_{R_{l m}}\left(u\left(\mathbf{x}_{l m}\right)+O(h)\right) \Delta \mathbf{G}_{I}^{h}\left(\mathbf{x}, \mathbf{x}_{l m}\right) d x d y \\
& =\sum_{R_{i j} \backslash R_{l m}}\left(u\left(\mathbf{x}_{i j}\right)+O(h)\right) O\left(h^{3}\right)+u\left(\mathbf{x}_{l m}\right) \iint_{R_{l m}} \Delta \mathbf{G}_{I}^{h}\left(\mathbf{x}, \mathbf{x}_{l m}\right) d x d y+O(h) \\
& =O(h)+u\left(\mathbf{x}_{l m}\right)+O(h)=u\left(\mathbf{x}_{l m}\right)+O(h),
\end{aligned}
$$

where $R_{i j}$ is the square centered at $\mathbf{x}_{i j}$. In the derivations above, we have used the fact that $\Delta \mathbf{G}_{I}^{h}\left(\mathbf{x}, \mathbf{x}_{l m}\right)=O(h)$ except for the square $R_{l m}$, and the area of $R_{i j}$ is $h^{2}$. This completes the proof.

Lemma 3.7. Let $C_{i j}^{I B}$ be the correction terms in the immersed boundary method (3.5), $v(s) \in C^{1}$ be defined in (3.1), and $\mathbf{G}_{I}^{h}\left(\mathbf{x}, \mathbf{x}_{l m}\right)$ be an interpolation function of $\mathbf{G}^{h}\left(\mathbf{x}_{i j}, \mathbf{x}_{l m}\right)$ that satisfies the conditions listed in Section $[3.2$. Then we have the following estimate:

$$
\sum_{i j} C_{i j}^{I B} \mathbf{G}^{h}\left(\mathbf{x}_{i j}, \mathbf{x}_{l m}\right) h^{2}=\int_{\Gamma} v(s) \mathbf{G}_{I}^{h}\left(\mathbf{X}(s), \mathbf{x}_{l m}\right) d s+O(h \log h) .
$$

Proof. We denote $h_{s}=\max \left\{\Delta s_{k}\right\} \sim h$, then

$$
\begin{aligned}
\sum_{i j} & C_{i j}^{I B} \mathbf{G}^{h}\left(\mathbf{x}_{i j}, \mathbf{x}_{l m}\right) h^{2} \\
& =\sum_{i j} \sum_{k=1}^{N_{b}} v_{k} \delta_{h}\left(x_{i}-X_{k}\right) \delta_{h}\left(y_{j}-Y_{k}\right) \Delta s_{k} \mathbf{G}^{h}\left(\mathbf{x}_{i j}, \mathbf{x}_{l m}\right) h^{2} \\
& =\sum_{k=1}^{N_{b}} v_{k} \Delta s_{k} \sum_{i j} \delta_{h}\left(x_{i}-x\right) \delta_{h}\left(y_{j}-y\right) \mathbf{G}_{I}^{h}\left(\mathbf{x}_{i j}, \mathbf{x}_{l m}\right) h^{2} \\
& =\sum_{k=1}^{N_{b}} v_{k} \Delta s_{k}\left(\mathbf{G}_{I}^{h}\left(\mathbf{X}_{k}, \mathbf{x}_{l m}\right)+E_{k}\right) .
\end{aligned}
$$

From the expression (4.2) and Lemma 4.1 in [3], we know that

$$
\left|E_{k}\right| \leq C h \sum_{\|\boldsymbol{\alpha}\|_{1}=1}\left|\partial^{\alpha} \mathbf{G}_{I}^{h}\left(\xi_{k}, \mathbf{x}_{l m}\right)\right|
$$

The summation $\sum_{k=1}^{N_{b}} v_{k} \Delta s_{k}\left(\mathbf{G}_{I}^{h}\left(\mathbf{X}_{k}, \mathbf{x}_{l m}\right)+E_{k}\right)$ is the composite trapezoidal rule for the line integral. We divide the summation into three groups, one with the summation of $k$ that $\left\|\mathbf{X}_{k}-\mathbf{x}_{l m}\right\|_{2} \geq \sqrt{h}$, one with $h \leq\left\|\mathbf{X}_{k}-\mathbf{x}_{l m}\right\|_{2} \leq \sqrt{h}$, and 
the other is for the remaining $k$ 's. The contributions from the boundary points are split as half and half for each group. For the first two groups, we have

$\sum_{\left\|\mathbf{X}_{k}-\mathbf{x}_{l m}\right\|_{2} \geq h}{ }^{\prime \prime} v_{k} \Delta s_{k}\left(\mathbf{G}_{I}^{h}\left(\mathbf{X}_{k}, \mathbf{x}_{l m}\right)+E_{k}\right)=\int_{\Gamma-\Gamma_{\mathbf{x}_{l m}}^{h}} v(s) \mathbf{G}_{I}^{h}\left(\mathbf{X}(s), \mathbf{x}_{l m}\right) d s+E_{h}^{1}$,

where" " means the coefficients are half at the two boundary points, and $\Gamma_{\mathbf{x}_{l m}}^{h}$ is the part of the interface that intersects the circle centered at $\mathbf{x}_{l m}$ with radius $h$. For the part $\left\|\mathbf{X}_{k}-\mathbf{x}_{l m}\right\|_{2} \geq \sqrt{h}$, we have

$$
\left|E_{h}^{1}\right| \leq C h_{s}^{2} \max _{k} \frac{1}{\left(\left\|\mathbf{X}_{k}-\mathbf{x}_{l m}\right\|_{2}+h\right)^{2}} \leq C h_{s}^{2} \frac{1}{(\sqrt{h}+h)^{2}} \leq C h,
$$

due to the second order partial derivatives of $\mathbf{G}_{I}^{h}\left(\mathbf{X}(s), \mathbf{x}_{l m}\right)$ and the first order derivatives of $E_{k}$.

For the part $h \leq\left\|\mathbf{X}_{k}-\mathbf{x}_{l m}\right\|_{2} \leq \sqrt{h}$, the error estimate is a little bit tricky. From the error estimate of the trapezoidal rule in each interval, we have

$$
\begin{aligned}
\left|E_{h}^{1}\right| & \leq \frac{h_{s}^{2}}{12} \sum_{k} h_{s} \sum_{\|\boldsymbol{\alpha}\|_{1}=2}\left|\partial^{\alpha} \mathbf{G}_{I}^{h}\left(\xi_{k}, \mathbf{x}_{l m}\right)\right| \leq C h_{s}^{2} \sum_{k} \frac{h_{s}}{\left.\left(\| \xi_{k}-\mathbf{x}_{l m}\right) \|_{2}+h\right)^{2}} \\
& \leq C h_{s}^{2} \int_{h_{s}}^{\sqrt{h_{s}}} \frac{d r}{(r+h)^{2}} \leq C h_{s}^{2} \int_{h_{s}}^{\sqrt{h_{s}}} \frac{d r}{r^{2}} \leq C h .
\end{aligned}
$$

For the last group, we have

$$
\begin{aligned}
\sum_{\left\|\mathbf{X}_{k}-\mathbf{x}_{l m}\right\|_{2} \leq h}{ }^{\prime \prime} v_{k} \Delta s_{k}\left(\mathbf{G}_{I}^{h}\left(\mathbf{X}_{k}, \mathbf{x}_{l m}\right)+E_{k}\right) & =\int_{\Gamma_{\mathbf{x}_{l m}}^{h}} v(s) \mathbf{G}_{I}^{h}\left(\mathbf{X}(s), \mathbf{x}_{l m}\right) d s+E_{h}^{2} \\
& \leq \max _{s} v(s) h \log h+E_{h}^{2}
\end{aligned}
$$

from the estimate of $\mathbf{G}_{I}^{h}\left(\mathbf{X}(s), \mathbf{x}_{l m}\right)$ in (3.16). For the error term $E_{h}^{2}$, since the length of the integral is $O(h)$, we have

$$
\left|E_{h}^{2}\right| \leq C h^{2} h \max _{\mathbf{X}_{k}} \frac{1}{\left(\left\|\mathbf{X}_{k}-\mathbf{x}_{l m}\right\|_{2}+h\right)^{2}} \leq C h^{3} \frac{1}{(h+h)^{2}} \leq C h .
$$

This completes the proof of the lemma.

Now we are ready to prove the main result of the paper.

Theorem 3.8. Let $u(x, y)$ be the solution to (3.1) and $\mathbf{U}$ is the solution vector obtained from the immersed boundary method (3.5) using a discrete delta function. Then $\mathbf{U}$ is first order accurate with a logarithm factor in the $L^{\infty}$ norm, that is,

$$
\left|E_{i j}\right| \leq C h \log h, \quad i, j=1,2, \cdots, n-1 .
$$


Proof. Consider the error at a grid point $E_{l m}$, if $\mathbf{x}_{l m}$ is close to the interface, that is, $\operatorname{dist}\left(\Gamma, \mathbf{x}_{l m}\right) \leq W h$, we have

$$
\begin{aligned}
E_{l m} & =\left(\left(A_{h}\right)^{-1} \mathbf{T}^{I B}\right)_{l m} \\
& =\left(\left(A_{h}\right)^{-1} \mathbf{T}_{r e g}^{I B}\right)_{l m}+\left(\left(A_{h}\right)^{-1} \mathbf{T}_{i r r}^{I B}\right)_{l m} \\
& =O\left(h^{2}\right)+\left(\left(A_{h}\right)^{-1} \mathbf{T}_{i r r}^{I B}\right)_{l m} \\
& =\sum_{\operatorname{dist}\left(\mathbf{x}_{i j}, \Gamma\right) \leq W h}\left(h^{2} T_{i j}\left(A_{h}\right)^{-1} \mathbf{e}_{i j} \frac{1}{h^{2}}\right)_{l m}+O\left(h^{2}\right) \\
& =\sum_{\operatorname{dist}\left(\mathbf{x}_{i j}, \Gamma\right) \leq W h} h^{2}\left(\Delta_{h} u\left(x_{i}, y_{j}\right)-C_{i j}^{I B}\right) \mathbf{G}^{h}\left(\mathbf{x}_{i j}, \mathbf{x}_{l m}\right)+O\left(h^{2}\right) \\
& =\sum_{i j} h^{2} \Delta_{h} u\left(x_{i}, y_{j}\right) \mathbf{G}^{h}\left(\mathbf{x}_{i j}, \mathbf{x}_{l m}\right)-\sum_{i j} h^{2} C_{i j}^{I B} \mathbf{G}^{h}\left(\mathbf{x}_{i j}, \mathbf{x}_{l m}\right)+O\left(h^{2}\right) \\
& \left.=\left(\int_{\Gamma} v(s) \mathbf{G}_{I}^{h}\left(\mathbf{X}(s), \mathbf{x}_{l m}\right) d s-\sum_{k} v_{k} \mathbf{G}_{I}^{h}\left(\mathbf{X}_{k}\right), \mathbf{x}_{l m}\right) \Delta s_{k}\right)+O(h) \\
= & O(h \log h),
\end{aligned}
$$

after we apply Lemma 3.6 and Lemma 3.7. Note that, in the expansion of the summation from $\operatorname{dist}\left(\mathbf{x}_{i j}, \Gamma\right) \leq W h$ to all interior grid points, we have used the fact that $\Delta_{h} u\left(x_{i}, y_{j}\right)=O\left(h^{2}\right)$ and $C_{i j}^{I B}=0$ when $\operatorname{dist}\left(\mathbf{x}_{i j}, \Gamma\right)>W h$. If $\operatorname{dist}\left(\Gamma, \mathbf{x}_{l m}\right)>$ $W h$, the proof above is still valid except that we are not going to have the singular integration. Thus, we do not need to have the $\log h$ factor. This means that for the IB method, the larger errors often occur near or on the interface.

\section{Conclusions And Discussions}

We give a convergence proof of the immersed boundary (IB) method in the $L^{\infty}$ norm. The key of the proof is to establish a connection between the discrete Green function and a continuous one with the same boundary conditions. We show that the IB method is indeed first order accurate with a $\log h$ factor if a reasonable discrete delta function is used. The reasonable conditions are the following: the discrete delta function should satisfy the zeroth moment condition (summation equals to the unity); with a local support (non-zero only in a small band of the interface with band width $W h$ ); and the interface does not intersect the boundary. The conclusion of the first order convergence should be applicable to other linear boundary conditions in addition to the Dirichlet type as long as the method of images applies. Furthermore, since quite a few Stokes and Navier-Stokes solvers such as the projection method, stream-vorticity formulations use fractional time splitting methods and Poisson/Helmholtz equations, that is, at each time step, the solvers involve solving several Helmholtz/Poisson equations. Thus the analysis in this manuscript for a single scalar equation can be applied.

The paper's result about the convergence of the IB method applies to any moment-preserving discrete delta functions. The first order convergence is guaranteed for any discrete delta function that satisfies the zeroth moment condition with a local support. 
On the other hand, whether the local truncation errors always behave like $O(1 / h)$ depends on several factors. Note that most moment-preserving discrete delta functions in the literature are derived for accurate line/surface integrals, but not for pointwise discretizations. Thus, we believe that for general interfaces, the local truncation errors near/on the interface do behave like $O(1 / h)$ for most of the discrete delta functions that have appeared in the literature. Some sophisticated discrete delta functions such as the one obtained from the source removal technique in 14 are a few exceptions.

\section{Appendix A. The COnstruction of An interpolation FunCtion of THE DiscRETE GREEN FUNCTION}

We construct an interpolation function from the grid function $\Delta \mathbf{G}_{I}^{h}\left(\mathbf{x}_{i j}, \mathbf{x}_{l m}\right)$ to the discrete Green function $\Delta \mathbf{G}_{I}^{h}\left(\mathbf{x}, \mathbf{x}_{l m}\right)$ that satisfies the conditions listed on page 1178 .

Let $\phi(x, y)$ be the function that satisfies the following conditions:

$$
\Delta \mathbf{G}_{I}^{h}\left(\mathbf{x}, \mathbf{x}_{l m}\right)=\delta_{h}(x, y), \quad \iint_{l m} \delta_{h}(x, y) d x d y=\frac{1}{h^{2}} .
$$

- $\Delta \mathbf{G}_{I}^{h}\left(\mathbf{x}, \mathbf{x}_{l m}\right)$ takes values of $\mathbf{G}_{I}^{h}\left(\mathbf{x}_{i j}, \mathbf{x}_{l m}\right)$ at four corners and the center.

In other words, we choose the source term and the boundary condition so that the two conditions above are satisfied.

Then, we construct the interpolation function $\mathbf{G}_{I}^{h}\left(\mathbf{x}, \mathbf{x}_{l m}\right)$ on other squares from the values of $\Delta \mathbf{G}_{I}^{h}\left(\mathbf{x}, \mathbf{x}_{l m}\right)$ using $\mathbf{Q}_{k}(K) \in C^{1}(\Omega)$, where $\mathbf{Q}_{k}$ is defined as the finite element space

$$
Q^{k}(K)=\left\{v(x, y), \quad \text { on each } R_{\hat{i} \hat{j}}, v(x, y)=\sum_{i=0, j=0}^{k} \beta_{i j} x^{i} y^{j}, \quad v(x, y) \in C^{1}(\Omega)\right\}
$$

over a quadrilateral mesh $K, R_{\hat{i} \hat{j}}$ is a square of $h$ by $h$. In the paper [23], the author has proposed a system to construct $C^{1}-Q^{k}$ finite element spaces on quadrilaterals meshes.

We have implemented and tested the interpolation function $\mathbf{G}_{I}^{h}\left(\mathbf{x}, \mathbf{x}_{l m}\right)$ of $Q^{5}(K) \in C^{1}(\Omega)$, which is third order accurate $(k=3)$. As usual, we just need to construct a shape function over a unit square. The total degree of freedom of $Q^{5}(K) \in C^{1}(\Omega)$ is 36 . In our construction, we specify the values of $v, v_{x}, v_{y}, v_{x x}$, $v_{x y}, v_{y y}$, which imposes 24 constraints. To keep the continuity of the solution and first order partial derivatives, for example, along the side $x=0$, we impose the coefficients of $x y^{5}$ and $x y^{4}$ to be zero. That is, there are two constraints along each side, which add an additional 8 constraints. Using the undetermined coefficient method for the 36 constraint with the 32 conditions, we have a system of equations with 36 unknowns and 32 equations (constraints). The condition number of the coefficient matrix, the ratio of the largest and smallest non-zero singular values is $8.9851 \times 10^{3}$, which indicates the matrix has full row rank and the system of equations has an infinite number of solutions. We suggest choosing the SVD solution as the interpolation function.

To see that the interpolation function is in $C^{1}$, we use the side $x=0$ as an example. Along this side, the function is a fifth polynomial, which is uniquely 


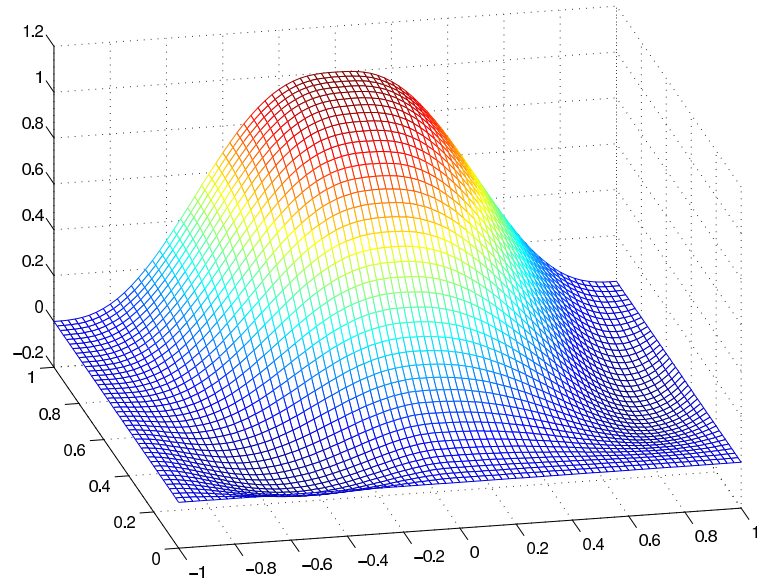

Figure 3. A plot of the shape function in $Q^{5}(K) \in C^{1}(\Omega)$ at two neighboring squares.

determined by its values, the first and second order derivatives at two points. Thus the interpolation function and its tangential derivative are continuous along this side. The normal derivative is in general still a fifth polynomial of $y$ along $x=0$. After we impose the constraints that its coefficients of $x y^{5}$ and $x y^{4}$ to be zero, it becomes a cubic polynomial which is uniquely determined by its values $\left(v_{x}\right)$ and tangential derivatives $\left(v_{x y}\right)$ at two points. In Figure 3, we show a plot of such an interpolation function in two neighboring squares with a function value at one corner being a unit while others and all the derivatives are zero.

A.1. Numerical verification of (3.15) and (3.16). We also verify the inequalities (3.15) and (3.16) numerically. Without loss of generality, we solve the discrete Laplacian on the square $[-1,1] \times[-1,1]$ using different meshes $m=n=$ $32,64,128,256,512$, and 1024. Since the solution is symmetric with $x$ and $y$, we just check $G_{I}^{h}, \frac{\partial G_{I}^{h}}{\partial x}$, and $\frac{\partial^{2} G_{I}^{h}}{\partial x^{2}}$. We check the inequalities of (3.15) and (3.16) at all the grid points and find the closest differences in the following matrix:

$$
-\left[\begin{array}{llllll}
0.2502 & 0.2501 & 0.2500 & 0.2500 & 0.2500 & 0.2500 \\
0.7032 & 0.7051 & 0.7061 & 0.7066 & 0.7068 & 0.7070 \\
0.5188 & 0.5093 & 0.5046 & 0.5023 & 0.5011 & 0.5006
\end{array}\right] .
$$

We can see that all the inequalities (3.15) for $G_{I}^{h}$, and (3.16) for $\frac{\partial G_{I}^{h}}{\partial x}$, and $\frac{\partial^{2} G_{I}^{h}}{\partial x^{2}}$ with $C=1$ are true. We also verify the inequalities of (3.15) and (3.16) graphically in Figure 4 (b)-(d) by plotting the differences of the quantities between the leftand right-hand sides of (3.15) and (3.16). 
(a)

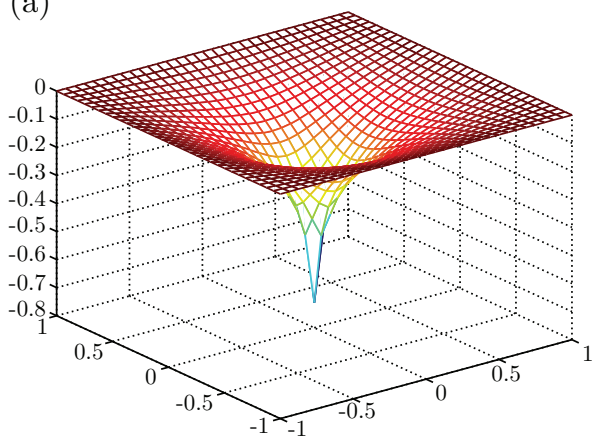

(c)

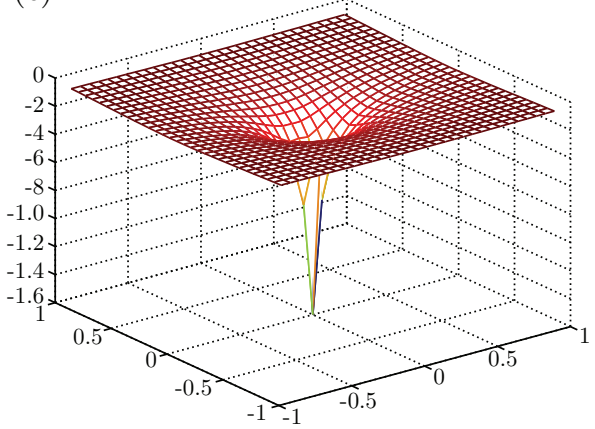

(b)

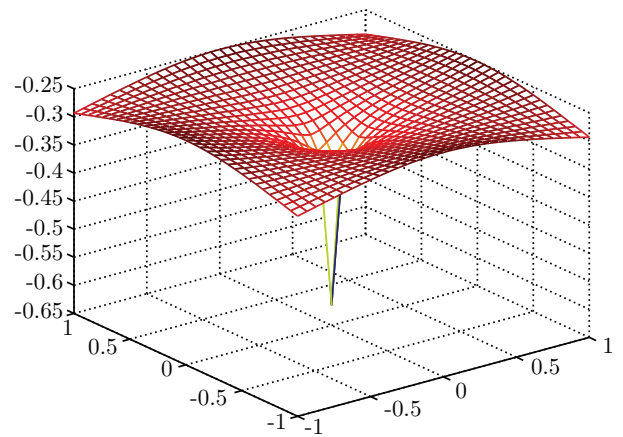

(d)

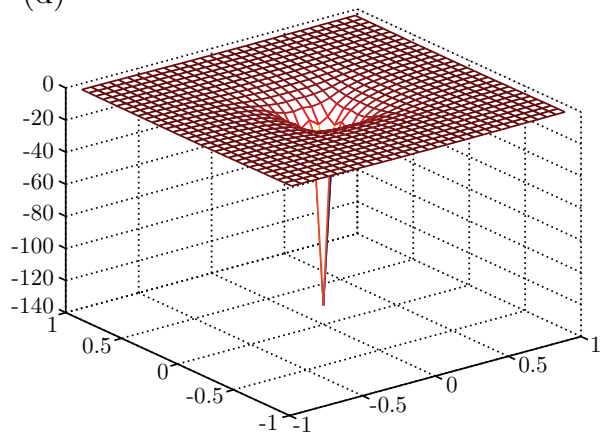

Figure 4. Plot (a), a discrete Laplacian with a 32 by 32 mesh. Plot (b), the differences of the quantities between the left- and right-hand sides of (3.15) for $G_{I}^{h}$; Plot (c)-(d), the differences of the quantities between the left- and right-hand sides of (3.16) for $\frac{\partial G_{I}^{h}}{\partial x}$ and $\frac{\partial^{2} G_{I}^{h}}{\partial x^{2}}$ with $C=1$, respectively.

\section{Appendix B. A numerical EXAmple}

We use a numerical example to show that the immersed boundary method is indeed first order accurate and its local truncation error is of order $O(1 / h)$. The differential equation is

$$
\Delta u(x, y)=\int_{\Gamma} 2 \delta(x-X(s)) \delta(y-Y(s)) d s, \quad-1<x, y<1,
$$

where the interface $\Gamma$ is the circle $r=1 / 2$, where $r=\sqrt{x^{2}+y^{2}}$. The function

$$
u(x, y)= \begin{cases}1 & \text { if } r \leq \frac{1}{2} \\ 1+\log (2 r) & \text { if } r>\frac{1}{2}\end{cases}
$$

satisfies the PDE. This example is taken from [11. We use the IB method to solve the PDE with the Dirichlet boundary condition given by (B.2). In Table 1, we list the results of a grid refinement analysis. In Table 1, the number $N$ is the number of grid lines in the $x$ and $y$ directions. The interface $r=1 / 2$ is discretized by $X_{k}=0.5 \cos (2 k \pi / N) Y_{k}=0.5 \sin (2 k \pi / N), k=0,1, \cdots, N-1$. 
TABLE 1. A grid refinement analysis of the IB method using the discrete cosine and hat delta functions.

\begin{tabular}{||c||c|c|c||c|c|c||}
\hline \hline$N$ & $\left\|E^{\cos }\right\|_{\infty}$ & order $^{\cos }$ & $\left\|T^{\cos }\right\|_{\infty}$ & $\left\|E^{\text {hat }}\right\|_{\infty}$ & order $^{\text {hat }}$ & $\left\|T^{\text {hat }}\right\|_{\infty}$ \\
\hline 20 & $5.721710^{-2}$ & & 17.3534 & $2.172410^{-2}$ & & 9.1327 \\
\hline 40 & $2.722610^{-2}$ & 1.0715 & 34.9848 & $9.993310^{-3}$ & 1.1202 & 20.6630 \\
\hline 80 & $1.339910^{-2}$ & 1.0228 & 69.9772 & $5.276110^{-3}$ & 0.9215 & 43.5721 \\
\hline 160 & $6.734010^{-3}$ & 0.9926 & 139.254 & $4.536510^{-3}$ & 2.1789 & 117.289 \\
\hline 320 & $3.351010^{-3}$ & 1.0066 & 279.483 & $1.885310^{-3}$ & 1.2667 & 195.664 \\
\hline 640 & $1.673710^{-3}$ & 1.0018 & 556.360 & $1.198510^{-3}$ & 0.6536 & 495.791 \\
\hline 1280 & $8.466310^{-4}$ & 0.88326 & 1122.01 & $5.402110^{-4}$ & 1.1497 & 892.463 \\
\hline
\end{tabular}

In the second and fifth columns of Table 1, we show the $L^{\infty}$ errors of the computed solution of the IB method using the discrete cosine and hat delta functions, respectively. The third and sixth columns show the approximate convergence order using the two consecutive errors. We see clearly first order convergence. In the fourth and seventh columns we show the local truncation errors in the $L^{\infty}$ norm. We see that the largest local truncation error increases in the order of $O(1 / h)$.

\section{ACKNOWLEDGMENTS}

The author acknowledge the support from the CNSF grants 11371199, 11071123, 11161036, and Jiangsu special visiting professorship grant in China. The author is grateful for many valuable suggestions and comments from Dr. J. Thomas Beale of Duke University and Dr. Kazufumi Ito of North Carolina State University. The author would also like to thank Fei Yu whose master thesis [22] is about constructing the interpolation functions using $C^{1}-Q^{5}, C^{2}-Q^{7}$ and other elements over rectangular meshes.

\section{REFERENCES}

[1] J. Adams, P. Swarztrauber, and R. Sweet, Fishpack: Efficient Fortran subprograms for the solution of separable elliptic partial differential equations. http://www.netlib.org/fishpack/.

[2] J. Thomas Beale and Anita T. Layton, On the accuracy of finite difference methods for elliptic problems with interfaces, Commun. Appl. Math. Comput. Sci. 1 (2006), 91-119 (electronic), DOI 10.2140/camcos.2006.1.91. MR2244270 (2009d:35047)

[3] R. P. Beyer and R. J. LeVeque, Analysis of a one-dimensional model for the immersed boundary method, SIAM J. Numer. Anal. 29 (1992), no. 2, 332-364, DOI 10.1137/0729022. MR.1154270(93a:65123)

[4] K.-Y. Chen, K.-A. Feng, Y. Kim, and M.-C. Lai, A note on pressure accuracy in immersed boundary method for stokes flow, J. Comput. Phys., 230:4377-4383, 2011.

[5] Lawrence C. Evans, Partial Differential Equations, Graduate Studies in Mathematics, vol. 19, American Mathematical Society, Providence, RI, 1998. MR 1625845 (99e:35001)

[6] K. Gürlebeck and A. Hommel, On finite difference potentials and their applications in a discrete function theory, Math. Methods Appl. Sci. 25 (2002), no. 16-18, 1563-1576, DOI 10.1002/mma.389. Clifford analysis in applications. MR.1949515 (2004a:39037)

[7] W. Hackbusch, Elliptic Differential Equations: Theory and Numerical Treatment, Springer Series in Computational Mathematics, vol. 18, Springer-Verlag, Berlin, 1992; Translated from the author's revision of the 1986 German original by Regine Fadiman and Patrick D. F. Ion. MR 1197118 (94b:35001) 
[8] H. Hasimoto, On the periodic fundamental solutions of the Stokes' equations and their application to viscous flow past a cubic array of spheres, J. Fluid Mech. 5 (1959), 317-328. MR.0102285 (21 \#1078)

[9] N. V. Krylov, Lectures on Elliptic and Parabolic Equations in HÖlder Spaces, Graduate Studies in Mathematics, vol. 12, American Mathematical Society, Providence, RI, 1996. MR.1406091 (97i:35001)

[10] Gregory F. Lawler, Intersections of Random Walks, Probability and its Applications, Birkhäuser Boston Inc., Boston, MA, 1991. MR1117680 (92f:60122)

[11] Randall J. LeVeque and Zhi Lin Li, The immersed interface method for elliptic equations with discontinuous coefficients and singular sources, SIAM J. Numer. Anal. 31 (1994), no. 4, 1019-1044, DOI 10.1137/0731054. MR.1286215 (95g:65139)

[12] Zhilin Li, The immersed interface method: A numerical approach for partial differential equations with interfaces, ProQuest LLC, Ann Arbor, MI, 1994. Thesis (Ph.D.)-University of Washington. MR2691718

[13] Zhilin Li and Kazufumi Ito, The Immersed Interface Method: Numerical Solutions of PDEs Involving Interfaces and Irregular Domains, Frontiers in Applied Mathematics, vol. 33, Society for Industrial and Applied Mathematics (SIAM), Philadelphia, PA, 2006. MR2242805 (2007g:65005)

[14] Zhilin Li, Wei-Cheng Wang, I-Liang Chern, and Ming-Chih Lai, New formulations for interface problems in polar coordinates, SIAM J. Sci. Comput. 25 (2003), no. 1, 224-245, DOI 10.1137/S106482750139618X. MR2047203 (2005e:65167)

[15] Yoichiro Mori, Convergence proof of the velocity field for a Stokes flow immersed boundary method, Comm. Pure Appl. Math. 61 (2008), no. 9, 1213-1263, DOI 10.1002/cpa.20233. MR2431702 (2009j:35274)

[16] Charles S. Peskin, Numerical analysis of blood flow in the heart, J. Computational Phys. 25 (1977), no. 3, 220-252. MR0490027 (58 \#9389)

[17] C. S. Peskin and D. M. McQueen, A general method for the computer simulation of biological systems interacting with fluids, Symposia of the Society for Experimental Biology, 49:265, 1995.

[18] V. Rutka, Immersed Interface methods for elliptic boundary value problems. PhD thesis, University of Kaiserslautern, 2005.

[19] Vidar Thomée, Discrete interior Schauder estimates for elliptic difference operators, SIAM J. Numer. Anal. 5 (1968), 626-645. MR0238505 (38 \#6781)

[20] Anna-Karin Tornberg and Björn Engquist, Numerical approximations of singular source terms in differential equations, J. Comput. Phys. 200 (2004), no. 2, 462-488, DOI 10.1016/j.jcp.2004.04.011. MR2095274 (2005e:65204)

[21] Björn Engquist, Anna-Karin Tornberg, and Richard Tsai, Discretization of Dirac delta functions in level set methods, J. Comput. Phys. 207 (2005), no. 1, 28-51, DOI 10.1016/j.jcp.2004.09.018. MR.2143581 (2006b:65081)

[22] F. Yu, Construction of $C^{1}-Q^{5}$ and $C^{2}-Q^{7}$ finite elements on $2 D$ rectangular meshes, North Carolina State University, MS. Thesis, 2012.

[23] Shangyou Zhang, On the full $C_{1}-Q_{k}$ finite element spaces on rectangles and cuboids, Adv. Appl. Math. Mech. 2 (2010), no. 6, 701-721. MR2719052(2011i:65230)

Center for Research in Scientific Computation (CRSC) and Department of Mathematics, North Carolina State University, Raleigh, North Carolina 27695 - and guest professor of School of Mathematical Sciences, Nanjing Normal University

E-mail address: zhilin@math.ncsu.edu 